\title{
SCATTERING MODEL FOR A COUPLE OF BUILDINGS IN SAR IMAGES
}

\author{
Alessio Ciotola, Gerardo Di Martino, Antonio Iodice, Daniele Riccio, Giuseppe Ruello \\ University of Naples Federico II, Via Claudio 21, 80125, Naples, Italy
}

\begin{abstract}
Data acquired by the last generation of SAR sensors over urban areas, thanks to high resolution, can reveal the presence and main features of buildings. Unfortunately geometric distortions introduced by system geometry and signal multiple reflections make SAR images very complex so we need a scattering model supporting both the visual inspection and the processing for information retrieval. In this paper we propose a scattering model able to describe the SAR response in terms of reflectivity map when a couple of buildings compose the scene. The model is based on Kirchhoff approximation and focuses on multiple reflections phenomenon which produce some linear bright structures in SAR images. A comparison between a real SAR image and a simulatation applying the model is reported.
\end{abstract}

Index Terms - SAR, urban areas, electromagnetic scattering

\section{INTRODUCTION}

The interpretation of a single SAR image over urban areas is a very difficult task. Although the high resolution of new sensors as TerraSAR-X, COSMO-Sky/Med and the future space mission Sentinel 1 allows to distinguish different man-made structures, distortions introduced by the geometry of the system together with mutual interaction among buildings distributed over the scene, influence the complexity of SAR image, which is very difficult to interpret and to manage by automatic algorithms. In order to better understand such images and for the development of simulators, we need some building scattering model expressed in closed form and characterized by a few parameters [1]-[3]. These characteristics make the model more effective if used for information retrieval or object detection in application scenarios with stringent time requirement, for example in case of damage assessment after natural disasters or in the field of military surveillance.

Our effort points to extend and generalize our previous work in which we introduced a scattering model describing the SAR response to an electromagnetic isolated building in term of RCS contributions [1]. In this paper we propose an innovative scattering model focusing on the electromagnetic

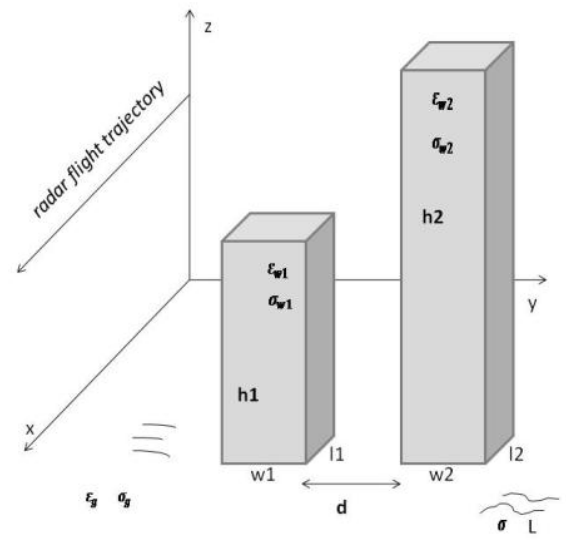

Fig. 1: representation of the considered scenario with all possible parameters included in the scattering model

interaction between a couple of close buildings. In particular the model tries to interpret bright linear structures in SAR images deriving from multiple reflections of radar signal among two consecutive built structures.

In section 2 we describe the fundamentals of the scattering model we propose, then in section 3 some experiments testing the effectiveness of the model are reported

\section{THE SCATTERING MODEL}

The presented model allows the description of the reflectivity map relative to a scene made by two flat roof structures surrounded by rough soil, both aligned with the flight direction. The considered scenario is depicted in Fig. 1 , in which two buildings are schematized as two flat blocks and all parameters involved are reported. Three kinds of parameters characterize the model: orbital data, geometric data and electromagnetic data. Orbital data include all parameters referring to the radar system and the acquisition geometry, i.e. the resolution in azimuth $(\Delta z)$ and range $(\Delta r)$, the carrier frequency $f_{0}$ and the radar signal incidence angle $\vartheta$. Geometric parameters refer to building's footprint dimensions $(w, l)$, their heights $(h)$, their reciprocal distance $(d)$ and to the statistic parameters describing the soil roughness i.e. the variance $(\sigma)$ and the correlation length $(L)$, Finally the electromagnetic data are conductivity and dielectric constants of soil, buildings roofs and walls 


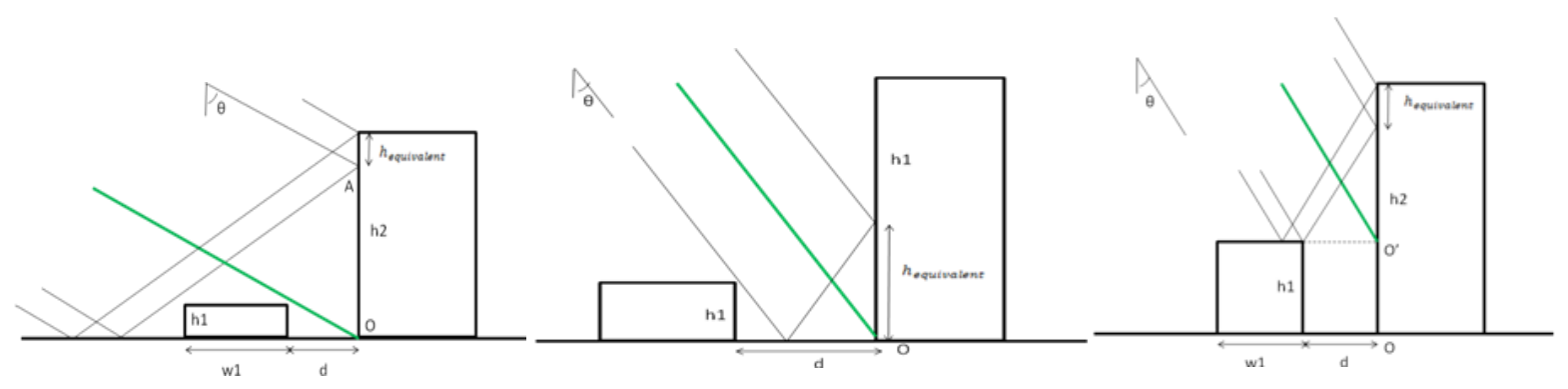

(a)

(b)

(c)

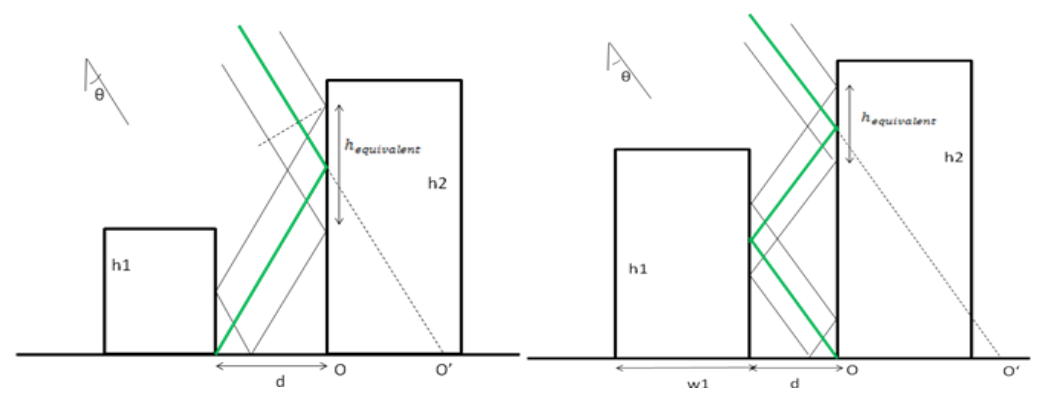

(d)

(e)

Fig. 2: representation of some possible multiple reflections: (a) 2-fold wall2-terrain between 1 and 2; (b) 2-fold wall 2-roof 1; (c) 2-fold wall 2-terrain before 1; (d) 4-fold reflection; (e) 6-fold reflection

$\left(\sigma_{g}, \varepsilon_{g}, \sigma_{w}, \varepsilon_{w}, \sigma_{r}, \varepsilon_{r}\right)$. The knowledge of such parameters supports the construction of a reflectivity map, intended as

The model starts from the hypothesis of a plane wave impinging the two buildings. The received radar signal is decomposed in two kind of contributions: single and multiple scattering. The first one refers to the signal that directly returns to the sensor from roofs, walls, and from soil; the second one concerns the multiple bounce through different objects. In turn, multiple bounce could be of a generic $n$-order, depending on how many reflections it experiments until it returns toward the sensor. Each contribution is characterized by a specific Radar Cross Section (RCS), whose superposition contributes to the formation of the resulting reflectivity map.

In Fig. 2 we show the graphic description of some examples of multiple reflection phenomenon of even order, up to 6th-order, considering the radar signal as constituted by optic rays. In Fig. 2 (a), (b) and (c) three different examples of double bounce reflection appear: while in the first two cases the signal bounces on building's wall and on the ground, in the third case a wall and a roof are involved. Then in Fig. 2 (d) and (e) the multiple bounces between building's walls are highlighted in the case of 4th and 6thorder, respectively. All rays involved in a multiple reflection, that travel the same path length, collapse in a single point in the reflectivity map and contribute all together to its RCS, resulting in a bright linear structure in the SAR image, which we call multiple reflection line. The positions of such lines in the SAR image are indirectly indicated by green rays in Fig. 2, i.e. the rays that travel the same track on the overall round trip, but with the same path length of interested multiple reflection. Hence, each multiple reflection seems to come from point indicated with $\mathrm{o}^{\prime}$ in Fig. 2.

The model provides RCS for a generic order $n$ of multiple reflection and is expressed in a closed form. It adopts Physical Optics (PO) approximation for the computation of backscattered field from soil and Geometric Optics (GO) for the one scattered by walls and roofs. Its expression is given by

$$
\begin{aligned}
\sigma^{o}= & 2 h_{\text {equivalent }}\left|S_{i j}\right|^{2} l \tan \vartheta \\
& \exp \left(-4 k^{2} \sigma^{2} \cos ^{2} \vartheta\right) \sum_{m=1}^{\infty} \frac{(2 k \sigma \cos \vartheta)^{2}}{m !} \frac{k^{2} L^{2}}{4 m}
\end{aligned}
$$

where $k$ is the wave number and $S_{i j}$ is the scattering matrix element. We refer to [2] for RCS formulas about single scattering contributions. Equation (1) is formally analogous to the RCS expression found for a dihedral structure formed by ground and a vertical wall of an isolate building [2]. However, there are two main differences: first of all now the analytical calculation of the scattering matrix $S_{i j}$ takes into 
account the order of reflection $n$ and the reflection coefficients of involved objects for the polarization configuration. For example in case of $\mathrm{HH}$ polarization the scattering matrix is

$$
\begin{aligned}
S_{H H}= & A_{H}\left[-2 R_{\perp G}(\vartheta) \cos \vartheta \cos 2 \varphi\right] \\
& +B_{H}\left\{\sin ^{2} \vartheta \sin 2 \varphi+R_{\| G}(\vartheta) \sin 2 \varphi\left(1+\cos ^{2} \vartheta\right)\right\}
\end{aligned}
$$

$R_{\perp G}$ and $R_{\| G}$ being the soil reflection coefficient while $A_{H}$ and $B_{H}$ have the following expressions:

$$
\begin{aligned}
& A_{H}=R_{\perp W} \cdot \cos ^{2} \vartheta \cdot \cos ^{2} \varphi-R_{\| W} \cdot \sin ^{2} \varphi \\
& B_{H}=\left(R_{\perp W}+R_{\| W}\right) \cdot \cos \vartheta \cdot \cos \varphi \cdot \sin \varphi
\end{aligned}
$$

where now $R_{\perp W}$ and $R_{\| W}$ refer to reflection coefficients of involved walls.

\section{TABLE I}

CONDITIONS FOR MULTIPLE REFLECTIONS EXISTENCE AND H-EQUIVALENT

\begin{tabular}{l|l}
\hline Exsistance conditions & h-equivalent \\
\hline $\begin{array}{l}h_{2}>(n / 2-1) d \operatorname{ctg}(\vartheta) \\
(n / 2-2) d \operatorname{ctg}(\vartheta)<h_{1}\end{array}$ & $\begin{array}{l}\text { if }\left(h_{2}-h_{1}\right)>d \operatorname{ctg}(\vartheta) \\
h_{\text {equivalent }}=2 \cdot\left(h_{1}\right. \\
<\left(\frac{n}{2}-1\right) d \operatorname{ctg}(\vartheta)\end{array}$ \\
$\begin{array}{l}\text { if }\left(h_{2}-h_{1}\right)<d \operatorname{ctg}(\vartheta) \\
\left.h_{\text {equivalent }}(\vartheta)\right) \\
=2 \cdot\left(h_{2}-(n / 2-1) d \operatorname{ctg}(\vartheta)\right)\end{array}$ \\
$\begin{array}{l}\text { if }\left(h_{2}+h_{1}\right)>(n-1) d \operatorname{ctg}(\vartheta) \\
h_{2}>(n / 2-1) d \operatorname{ctg}(\vartheta) \\
(n / 2-1) d \operatorname{ctg}(\vartheta)<h_{1} \\
<(n / 2) d \operatorname{ctg}(\vartheta)\end{array}$ & $\begin{array}{l}\text { if }\left(h_{2}+h_{1}\right)<(n-1) d \operatorname{ctg}(\vartheta) \\
h_{\text {equivalent }}=2 \cdot\left(h_{2}\right.\end{array}$ \\
& \\
\hline
\end{tabular}

Thus, while in case of isolated building the RCS is proportional to the entire building height, now not necessarilyy all the height is involved in the multiple reflection phenomenon but just part of it, so in the formula $h_{\text {equivalent }}$ appears, which depends on buildings geometric features, their distance $d$, the look angle $\vartheta$ and the order of reflection $n$ (see Fig. 2). In particular, we have individuated geometric conditions that, for every reflection order, verify the presence of multiple reflection. Such conditions are reported in Table 1 together with respective $h_{\text {equivalent }}$ value.

Concerning to multiple reflection line location, if $o$ is the ground-range coordinate of the wall's base, than its position in the reflectivity map has the coordinate

$$
o^{\prime}=o+\left(\frac{n}{2}-1\right) d
$$

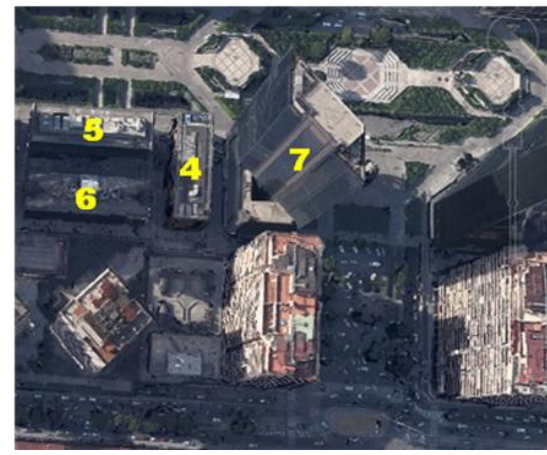

Fig. 3: aerial optic view of analyzed buildings

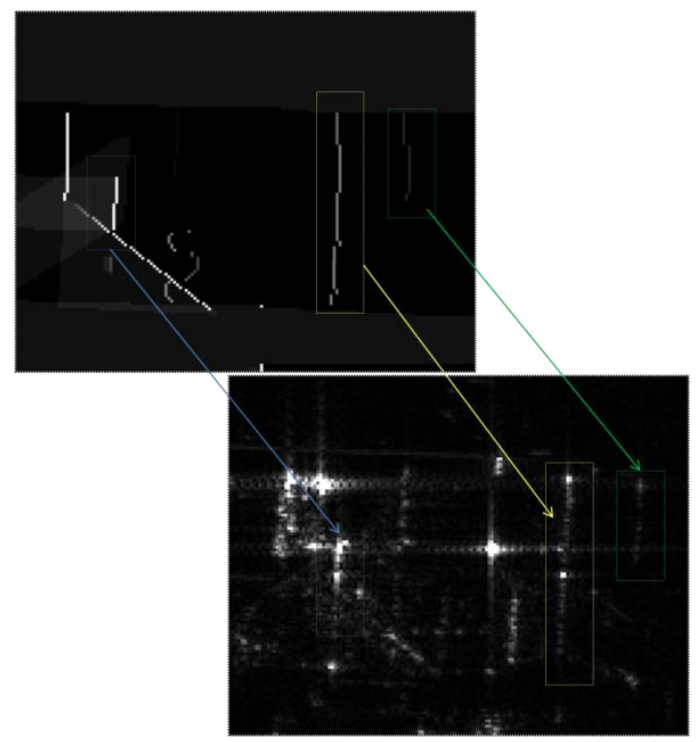

Fig. 4: at bottom the real SAR image of considered buildings; on top correspondent simulated image

as it could be simply demonstrated by geometric considerations.

\section{CASE STUDY}

In the following, we find an example in which we demonstrate the capability of the model to forecast the occurrence of a multiple reflections lines, but also their relative RCS values with respect to surrounding ones and their positions in the SAR image. The scattering model has been integrated in a SAR image simulator. The investigated site is the Business District Center of Naples, Italy. Such a site is particularly valuable for our scope, because it is crowded by tall and closely spaced buildings with a regular structure.

Hence, we expect that all hypotheses for the occurrence of multiple reflections are verified. In Fig. 3, an optical view of the investigated buildings is shown. They are identified by a yellow number for clearness. Then in the upper part of 
Fig. 4 we report the simulated image, in which multiple reflections that appear as bright linear structures are distinguishable; this, thanks to the property of multiple reflections to condense field energy from more rays in a single pixel. In the bottom of the same figure the real SAR image is presented. Correspondent lines of multiple reflections are highlighted in a rectangle and linked with colored arrows. The blue one refers to the ground-wall double bounce arising from the middle part of building 4 . Then the yellow one evidences the 4-fold reflection induced by the frontal walls of building 4 and 7 . Finally, the green rectangle contains the 6 -fold interaction between the same buildings. It is worthwhile to note that such a contribution is a shorter line respect to 4-fold one for the particular shape of building 7: in fact, it is composed by a block with parallelogram base superimposed on a rectangular parallelepiped. Therefore, the 6 -fold reflection appears just in the upper part, i.e. where the two walls are aligned.

Such an example points out how the proposed scattering model is able to forecasts structures actually visible in a real SAR image and its capability to locate them in the correct position.

\section{REFERENCES}

[1] G. Franceschetti, M. Migliaccio, D. Riccio, G. Schirinzi, "SARAS: A Synthetic Aperture Radar (SAR) Raw Signal Simulator", IEEE Transactions on Geoscience and Remote Sensing, GE-30, 110-123, January1992.

[2] G. Franceschetti, A. Iodice, D. Riccio, "A Canonical Problem in Electromagnetic Backscattering from Buildings", IEEE Transactions on Geoscience and Remote Sensing, GE-40, 17871801, August 2002.

[3] G. Franceschetti, A. Iodice, D. Riccio, G.Ruello, "SAR Raw Signal Simulation for Urban Structures", IEEE Transactions on Geoscience and Remote Sensing, GE-41, 1986-1995, September 2003. 